\title{
WHEN IS A KIRILLOV ORBIT A LINEAR VARIETY?
}

\author{
RAINER FELIX
}

\begin{abstract}
It is well known that a Kirillov orbit is a linear variety if and only if the corresponding irreducible representation is square integrable modulo its kernel ([1], Theorem 1.1). Now we give a new representation-theoretic criterion for a Kirillov orbit being a linear variety in terms of weak containment and tensor products of group representations.
\end{abstract}

Let $G$ be a nilpotent simply connected Lie group and $\mathbb{S}$ its Lie algebra. For $\pi \in \hat{G}$, the set of equivalence classes of irreducible unitary representations of $G$, let $\Omega_{\pi}$ be the corresponding Kirillov orbit in the dual space $\mathbb{E} *$ of $\mathbb{E}$. The orbit corresponding to the one-dimensional identity representation 1 of $G$ is $\{0\}$, and the orbit corresponding to the conjugate representation $\bar{\pi}$ of $\pi$ is $-\Omega_{\pi}$. For $\pi, \rho \in \hat{G}$ an element $\sigma \in \hat{G}$ is weakly contained in the tensor product $\pi \otimes \rho$ of $\pi$ and $\rho$ if and only if $\Omega_{\sigma} \subseteq \overline{\Omega_{\pi}+\Omega_{\rho}}$ [2, Lemma 2.1]. Therefore 1 is weakly contained in $\pi \otimes \bar{\pi}$ for every $\pi$ (compare $[4$, Lemma 1$])$. Now we investigate under which condition 1 is weakly contained in $\pi \otimes \bar{\rho}$ for $\rho \neq \pi$ (compare $[3,2])$.

THEOREM. The following properties of $\pi \in \hat{G}$ are equivalent:

(i) $\Omega_{\pi}$ is a linear variety;

(ii) if 1 is weakly contained in $\pi \otimes \bar{\rho}$ for $\rho \in \hat{G}$, then $\rho=\pi$.

Proof. Let $\Omega_{\pi}$ be a linear variety and $0 \in \overline{\Omega_{\pi}-\Omega_{\rho}}$. Then $\Omega_{\pi}=p+M$, where $p \in \mathscr{S F}^{*}$ and $M$ is a linear subspace of $\left.\&\right)^{*}$, which is invariant under the coadjoint representation. The quotient representation of the coadjoint representation in the quotient space $\mathbb{S S}^{*} / M$ is unipotent, consequently the orbits in $\mathbb{S}^{*} / M$ are closed. Thus $k\left(\Omega_{\rho}\right) \subseteq \mathbb{S}^{*} / M$ is closed, where $k: \mathbb{S}^{*} \rightarrow \mathbb{S}^{*} / M$ is the canonical mapping. By $0 \in \overline{\Omega_{\pi}-\Omega_{\rho}}$ we follow $k(p) \in k\left(\Omega_{\rho}\right)$, then $k(p)=k(q)$ with $q \in \Omega_{p}$, then $q \in p+$ $M=\Omega_{\pi}$.

Conversely, assuming that $\Omega_{\pi}$ is not a linear variety, we have to show that there exists an orbit $\Omega \neq \Omega_{\pi}$ with $0 \in \overline{\Omega_{\pi}-\Omega}$. By assumption, there are points $p, q \in \Omega_{\pi}$ such that the line segment $[p, q]$ joining $p$ and $q$ is not contained in $\Omega_{\pi}$. Let $\operatorname{Exp} \mathbf{R} X$, $X \in L\left(\&^{*}\right)$, be a one-parameter subgroup of the coadjoint group of $G$ with $(\operatorname{Exp} X) p=q$. Because $X$ is a nilpotent endomorphism, there is a greatest natural

Received by the editors December 24, 1981.

1980 Mathematics Subject Classification. Primary 22E25, 22D10.

Key words and phrases. Nilpotent Lie groups, tensor products of group representations, weak containment. 
number $n$ such that $X^{n} p \neq 0$. Let $m \leqslant n$ be the smallest number such that $\operatorname{Exp} \mathbf{R} X p$ $+F \subseteq \Omega_{\pi}, F$ being the linear hull of the vectors $X^{m+1} p, X^{m+2} p, \ldots, X^{n} p$ in (S)* (The linear subspace $F$ of $\&$ * can be regarded as the "flat part" of $\Omega_{\pi}$ along $\operatorname{Exp} \mathbf{R} X p$.) In view of $[p, q] \nsubseteq \Omega_{\pi}$ we conclude $m>1$. Thus we can find $\alpha, \beta \in \mathbf{R}$ and $f \in F$ such that the point $q:=(\operatorname{Exp} \alpha X) p+\beta X^{m} p+f$ does not belong to $\Omega_{\pi}$. Let $\Omega$ be the coadjoint orbit of $q$. The subspace $F$ of $\mathscr{S}^{*}$ is invariant under $X$. Therefore we can form the endomorphism $X_{F}$, associated with $X$, of the quotient space (S) $/ F$. In order to see that $0 \in \overline{\Omega_{\pi}-\Omega}$ it is enough to show that

$$
0 \in \overline{k(\operatorname{Exp} \mathbf{R} X p)-k(\operatorname{Exp} \mathbf{R} X q)}=\overline{\operatorname{Exp} \mathbf{R} X_{F} k(p)-\operatorname{Exp} \mathbf{R} X_{F} k(q)}
$$

in $\mathscr{S}^{*} / F, k: \mathbb{S}^{*} \rightarrow \mathbb{S}^{*} / F$ being the canonical mapping. Let us define the sequence

$$
x_{\nu}:=\left(\operatorname{Exp} \gamma_{\nu} X_{F}\right) k(p) \in \operatorname{Exp} \mathbf{R} X_{F} k(p)
$$

with $\gamma_{\nu}:=\left(\nu^{m}+m ! \beta\right)^{1 / m}$ and the sequence

$$
x_{\nu}^{\prime}:=\left(\operatorname{Exp}(\nu-\alpha) X_{F}\right) k(q) \in \operatorname{Exp} \mathbf{R} X_{F} k(q) .
$$

Then

$$
\begin{aligned}
x_{\nu}-x_{\nu}^{\prime} & =\sum_{r=0}^{m} \frac{\gamma_{\nu}^{r}-\nu^{r}}{r !} X_{F}^{r} k(p)-\beta X_{F}^{m} k(p) \\
& =\sum_{r=0}^{m-1} \frac{\gamma_{\nu}^{r}-\nu^{r}}{r !} X_{F}^{r} k(p)
\end{aligned}
$$

is a null sequence, because $\gamma_{\nu}^{r}-\nu^{r}$ is a null sequence in $\mathbf{R}$ for $r<m$.

COROLLARY. $\pi \in \hat{G}$ is square integrable modulo its kernel if and only if any element $\rho \in \hat{G}$, for which 1 is weakly contained in $\pi \otimes \bar{\rho}$, must be equal to $\pi$.

Maybe the assertion of this corollary remains true for more general locally compact groups.

ACKNOWLEDGEMENTS. The author is indebted to E. Kaniuth for the conjecture that all orbits are linear varieties iff for all $\pi, \rho \in \hat{G}$ the trivial representation 1 is weakly contained in $\pi \otimes \bar{\rho}$ only for $\pi=\rho$ (compare [4, Examples]).

\section{REFERENCES}

1. J. Brezin, Geometry and the method of Kirillov, Lecture Notes in Math., vol. 466, Springer-Verlag, Berlin-Heidelberg-New York, 1975, pp. 13-26.

2. R. Felix, Über Integralzerlegungen von Darstellungen nilpotenter Liegruppen, Manuscripta Math. 27 (1979), 279-290.

3. J. M. G. Fell, Weak containment and Kronecker products of group representations, Pacific J. Math. 13 (1963), 503-510.

4. E. Kaniuth, Weak containment and tensor products of group representations, Preprint.

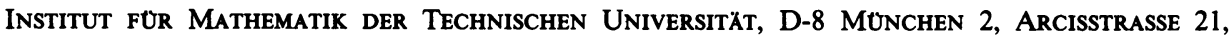
POSTFACH 2024 20, GeRmany 